(Aus dem physiologischen Laboratorium der Klinik für Geistes- und Nervenkrankheiten zu St. Petersburg. Vorsteher: Prof. W. v. Bechterew.)

\title{
Die Entwicklung der Pupillen- und anderer Augenreflexe bei neugeborenen Säugetieren.
}

Von

Sergius Michailow.

(Mit 1 Textfigur.)

In der letzten Zeit erfährt in der Physiologie die Lehre von dem Reflexakt bedeutende Umgestaltungen im Sinne einer Erweiterung dieses Begriffes und einer Unterordnung unter den Begriff des Reflexes solcher nervöser Akte und Reaktionen, die unlängst und mitunter auch noch jetzt als Prozesse betrachtet wurden und betrachtet werden, die zu einer ganz anderen Kategorie von Erscheinungen, zur Kategorie der psychischen Erscheinungen gehören. Dabei enthalten die Definitionen der reflektorischen Prozesse in sich oft den Begriff, dass diese Prozesse sich zum gegebenen Individuum von seinen Vorfahren vererben, und dass die betreffenden Individuen mit den Reflexen als mit schon fertigen und vollentwickelten Mechanismen und Reaktionen zur. Welt gelangen.

Diese Behauptungen wollte ich an der Entwicklung eines so klassischen und von allen anerkannten beständigen, einfachen und natürlichen Reflexes, wie es der Pupillenreflex ist, kontrollieren. Wie bekannt, kommen viele Säugetiere (Hund, Kaninchen und verschiedene andere) blind, mit geschlossenen Augen zur Welt und, naturgemäss, wäre für sie, solange sie die Augen nicht öffnen, das Vorhandensein des Pupillen- und anderer Augenreflexe ganz zwecklos. Meine Aufgabe besteht zunächst eben darin, festzustellen, ob die Pupille neugeborener (blind zur Welt kommender) auf Licht und ob sie auf Reizung des Halssympathicus gleich vom Moment der Geburt des Tieres an reagiert, und, wenn das nicht der Fall sein sollte, zu 
eruieren, wann dieser Reflex zur Entwicklung kommt. Späterhin gesellten sich zu dieser Grundaufgabe noch einige andere Fragen hinzu, von denen ich in den Protokollen der einzelnen Experimente Erwähnung tun werde." Die Beantwortung der aufgeworfenen einfachen Frage in Angriff zu nehmen war, natürlich, auch noch aus dem Grunde interessant, als in der ganzen riesigen, Hunderte von Arbeiten zählenden Literatur über den Pupillenreflex, soweit mir bekannt, noch nie die Frage nach der Entwicklung dieses Reflexes aufgeworfen worden ist.

Als Untersuchungsobjekt dienten neugeborene Junge trächtiger Hündinnen, die speziell zum Zwecke der genannten Untersuchungen am Laboratorium gehalten wurden; das Alter der neugeborenen Tiere konnte folglich genau festgestellt werden. An den jungen dem Experiment unterliegenden Hunden wurde ohne Anwendung irgend einer Narkose die allen bekannte Operation ausgeführt, welche zum Zwecke hat, den Nervus vagosympathicus auf dieser oder jener Seite des Halses auf Ligatur zu nehmen. Da aber noch Untersuchungen der Veränderung des Pupillendurchmessers unter dem Einfluss der Reizung des Halssympathicus bevorstanden, war es noch vor Ausführung der obengenannten Operation zur Auffindung des Nervus vagosympathicus notwendig, den Augapfel des dem Experiment unterliegenden und mit geschlossenen Augen zur Welt kommenden Tieres der Beobachtung zugänglich zu machen. Zu diesem Zwecke wurde eine radikale Operation - Abschneiden der oberen und unteren Augenlider beiderseits - ausgeführt, wonach die Augen ganz deutlich besichtigt werden konnten. Bei der Reizung des Halsvagosympathicus bloss der einen Körperseite hielt ich es in der grossen Mehrzahl der Fälle dennoch für zweckmässig, durch den Schnitt beide Augen zu öffnen, um immer das andere Auge zum Vergleich hinzuziehen zu können. Es ist wohl nicht überflüssig, hierbei zu bemerken, dass die bei Ausführung der genannten Operation auftretende Blutung immer leicht getilgt werden konnte durch Aufdrücken von mit $\mathrm{zu} 37$ bis $38^{\circ}$ C. erwärmter physiologiseher Kochsalzlösung oder mit Ringer-Locke'scher Lösung getränkten Wattestückchen. Infolge dieses Umstandes büssten die neugeborenen Tiere nicht ihre Kraft ein und wurden nicht schwach (wie bekannt, sind neugeborene und junge Tiere Blutungen gegenüber besonders empfindlich).

Nach der künstlichen Eröffnung der Augen wurde sofort und zunächst die Reaktion der Pupillen auf Licht, welches auf diese 
Weise zum erstenmal aufs Auge fiel, beobachtet, und nachdem mit Bestimmtheit das Vorhanden- oder Nichtvorhandensein des Pupillenreflexes auf Lichtreiz festgestellt worden war, wurden die Augen mit in einer der oben genannten Flüssigkeiten befeuchteten Wattestückchen zugedeckt.

Weiter wurde die oben beschriebene Operation am Halse zur Unterbindung einer der beiden Halsvagosympathici ausgeführt, welcher auch dann neben dem Ganglion cervicale inferius - etwas höher - durchschnitten wurde. Das Kopfende des durchschnittenen Nerven wurde mittelst des elektrischen Stromes eines Akkumulators, welcher mit einem Dubois - Reymond'schen Schlittenapparat und zwei aus Platin verfertigten, hakenförmig gebogenen Elektroden (auf die der Nerv gelegt wurde) in Verbindung stand, gereizt.

Nachdem auf solche Weise die Mitteilung der vorläufigen allgemeinen Bemerkungen erledigt ist, wollen wir direkt zu einer kurzen Beschreibung der Experimente selbst schreiten.

Wurf $I$, bestehend aus fünf Jungen von einem weiblichen. Hofhunde.

\section{Experiment am 24. Juni 1908.}

Hündchen I, geboren am 23. Juni 1908. Gewicht $250 \mathrm{~g}$, Länge $12 \mathrm{~cm}$. Die Augen sind durch den Schnitt eröffnet worden. Die Pupille ist so vergrössert (!), dass die Iris sie bloss als schmaler Reif (1 bis $2 \mathrm{~mm}$ ) umgibt. Auf Lichtreiz mittelst einer elektrischen Lampe und auch beim Einfallen von Sonnenstrahlen in die Augen (es war ein sonniger Tag) tritt nicht die geringste Verengerung der Pupille ein. Die Nickhaut (Membrana nictitans) ist erschlafft. Es wird unterbunden und durchschnitten der linke cervicale Nervus vagosympathicus. Bei seiner Reizung mittelst des elektrischen Stromes von der beweglichen Sekundärspirale eines $\mathrm{Dub}$ is Reymond'schen Apparates beim Rollenabstand von 18-15-1210-8-7-6-5 cm tritt gar kein Effekt im Sinne einer weiteren Vergrösserung der Pupille, Kontraktion der Nickhaut usw. ein alles bleibt in statu quo, als ob der Nerv gar nicht gereizt worden wäre.

Das Benetzen der Augäpfel mittelst der obengenannten physiologischen Lösungen sowie das Berühren mittelst eines Instrumentes oder Watte ruft Bewegungen des gereizten Augapfels (nach vorne und seitwärts) hervor; die Pupille bleibt aber auch dann unbeweglich. 


\section{Experiment am 25. Juni 1908.}

Junger Hund 2, geboren am 23. Juni 1908. Gewicht $320 \mathrm{~g}$, Länge $12 \mathrm{~cm}$. Die Augen sind durch den Schnitt eröffnet. Die Pupille ist verbreitert, wie auch bei dem gestrigen Hündehen; die Nickhaut ist ebenso erschlafft. Auf Lichtreiz trat kein Effekt ein; Reizung der Oberfläche der Augäpfel mittelst termischer und mechanischer Mittel gab das gleiche Resultat wie auch gestern. Reizung des rechten Nervus vagosympathicus mit Strömen verschiedener Stärke blieb obne Effekt.

\section{Experiment am 26. Juni 1908.}

Hündehen 3, geboren am 23. Juni 1908. Gewicht $300 \mathrm{~g}$, Länge $13 \mathrm{~cm}$. Die Augen sind durch den Schnitt eröffnet. Die Pupille ist verbreitert, wie bei den vorhergehenden Hündchen, und ebenso ist die Nickhaut erschlafft. Es wurde unterbunden und durchsehnitten der rechte cervicale Nervus vagosympathicus, nachdem es feststand, dass die Pupillen auf Lichtreiz nicht reagieren. Bei Reizung des zentralen Endstückes des durchschnittenen Nerven mittelst elektrischen Stromes von der Sekundärspirale eines $D$ ubois-Reymond'schen Apparates beim Rollenabstand von $15 \mathrm{~cm}$ bleibt die Pupille unbeweglich, die Nickhaut aber kontrahiert sich. Wiederholte Reizungen mit Strömen der gleichen Stärke sowohl als beim Rollenabstand von $12-10-8 \mathrm{~cm}$ hatten das gleiche Ergebnis.

\section{Experiment am 30. Juni 1908.}

Junger Hund 4, geboren am 23. Juni 1908. Gewicht $480 \mathrm{~g}$, Länge $18 \mathrm{~cm}$. Die Augen sind durch den Schnitt eröffnet. Die Pupille verengerte sich sofort bedeutend auf Licht. Es wurde unterbunden und durchschnitten der linke cervicale Nervus vagosympathicus. Bei dessen Reizung mit dem elektrischen Strome von einem DuboisReymond'schen Apparate beim Rollenabstand von $18 \mathrm{~cm}$ trat von seiten der Pupille und der Nickhaut ein vollständig deutlicher Effekt zutage, wie er unter analogen Bedingungen bei erwachsenen Tieren der gleichen Art beobachtet wird: die Pupille verbreitërte sich prompt und deutlich, die Nickhaut kontrahierte sich.

\section{Experiment am 9. Juli 1908.}

Junger Hund 5, geboren am 23. Juni 1908. Gewicht $675 \mathrm{~g}$, Länge $22 \mathrm{~cm}$. Die Augen begannen am 7. Juli 1908 sich zu öfnen, und am Experimenttag ist das rechte Ange noch nicht voll- 
ständig geöffnet, weil am lateralen Rande die Augenlider noch verwachsen sind, während das linke Auge schon in ganz normaler Weise offen ist. Die Lider werden an beiden Augen abgeschnitten. Die Pupillenreaktion auf Licht ist deutlich ausgesprochen. Es wurde unterbunden und durchschnitten der rechte Nervus vagosympathicus. Bei Reizung mittelst des elektrisehen Stromes von der Sekundärspirale eines Dubois-Reymond'schen Apparates beim Rollenabstand von $18 \mathrm{~cm}$ trat ein deutlicher Effekt im Sinne einer Verbreiterung der Pupille, Kontraktion der Nickhaut usw. ein.

Wurf II, bestebend aus fünf Jungen von einem weiblichen Hofbunde.

Experiment am 31. Juli 1908.

Hündchen 1, geboren am 28. Juli 1908. Gewicht $500 \mathrm{~g}$, Länge $16 \mathrm{~cm}$. Die Augen sind durch den Schnitt eröffnet worden. Die Pupille ist verbreitert, die Nickhaut ersehlafit. Es wurde unterbunden und durchschnitten der rechte Nervus vagosympathicus. Bei Reizung des zentralen Endes des durchschnittenen Nerven trat von seiten der Pupille beim Rollenabstand von $15-10-5 \mathrm{~cm}$ kein Effekt ein, während sich die Nickhaut hierbei deutlich kontrahierte.

\section{Experiment am 2. August 1908.}

Hündchen 2, geboren am 28. Juli 1908. Gewicht $550 \mathrm{~g}$, Länge $18 \mathrm{~cm}$. Die Augen sind durch Schnitt eröffnet worden. Die Pupille ist verbreitert und reagiert nicht auf Licht. Es wurde unterbunden und durchschnitten der linke Nervus vagosympathicus, wobei die Reizung des zentralen Endes bei einem Rollenabstand von 15-10$5 \mathrm{~cm}$ von gar keinem Effekt von seiten der Pupille begleitet war, während die Nickhaut sich hierbei sehr energisch kontrahierte. Darauf wurde ebenso unterbunden und durehschnitten der rechte Nervus vagosympathicus und sein zentraler Stumpf mit Strömen von derselben Stärke und mit genau dem gleichen Ergebnis gereizt.

\section{Experiment am 4. August 1908.}

Hündchen 3, geboren am 28. Juli 1908. Gewicht $725 \mathrm{~g}$, Länge $23 \mathrm{~cm}$. Die Augen sind durch den Schnitt eröffnet worden. Die Pupille kontrahierte sich auf Licht, aber uicht so prompt wie beim Hündehen I, 4. Darauf wurde der rechte Nervus vagosympathicus unterbunden und durchschnitten. Infolge starker Unruhe wurde das 
Tier für einige Zeit in Ruhe gelassen, und dann wurde der zentrale Stumpf des durchschnittenen Nerven gereizt, wobei beim Rollenabstand von $15 \mathrm{~cm}$ Verbreiterung der Pupille, Kontraktion der Nickhaut, Hervorwölben des Augapfels aus der Orbita, d. h. dessen Bewegung nach vorn aussen, eintrat. Alle diese Bewegungen sowohl als die ihnen entgegengesetzten nach Aufbören der Reizung erfolgen träge und ziemlich langsam. Der gleiche Effekt wurde auch bei wiederholentlichen Reizungen einige Male erbalten.

Das vierte Hündchen dieser Serie wurde zu Zwecken, die in vorliegender Arbeit angestrebt werden, nicht benutzt.

\section{Wurf III, bestehend aus vier Jungen von einem weiblichen Pudel.}

Experiment am 17. August 1908.

Hündchen 1, geboren in der Nacht vom 16. auf den 17. August 1908. Zur Zeit des Experimentes war es 15 Stunden alt. Gewicht $410 \mathrm{~g}$, Länge $15 \mathrm{~cm}$. Die Augen sind durch den Schnitt eröffnet worden. Die Pupille ist verbreitert, die Nickhaut erschlafft. Fis wurde unterbunden und nachher durchschnitten der rechte Nervus vagosympathicus. Bei Reizung seines zentralen Endes mittelst des elektrischen Stromes beim Rollenabstand von $15--10-8-5$ und sogar $2 \mathrm{~cm}$ trat von seiten der Pupille und der Nickhaut gar kein Effekt ein.

\section{Experiment am 23. August 1908.}

Hündchen 2, geboren in der Nacht vom 16. auf den 17. August 1908. Gewicht $600 \mathrm{~g}$, Länge $28 \mathrm{~cm}$. Die Augen sind durch den Schnitt eröffnet worden. Die Pupille kontrahierte sich prompt auf Licht. Es wurde unterbunden und durchschnitten der linke Nervus vagosympathicus, dessen zentraler Stumpf darauf mit dem elektrischen Strom beim Rollenabstand von $18 \mathrm{~cm}$ gereizt wurde. Als Folge dieser Reizung trat Verbreiterung der Pupille und Kontraktion der Nickhaut ein.

\section{Experiment am 25. August 1908.}

Hündchen 3, geboren in der Nacht vom 16. auf den 17. August 1908. Gewicht $680 \mathrm{~g}$, Länge $31 \mathrm{~cm}$. Die Augen durch den Schnitt eröffnet. Die Pupille verengerte sich prompt auf Lichteinfall. Es wurde unterbunden und durchsehnitten der rechte Nervus vagosympathicus. Reizung seines zentralen Stumpfes führte zu deutlicher und energischer 
Verbreiterung der Pupille, Kontraktion der Nickhaut und Bewegung des Augapfels nach aussen, d. h. seinem Hervorrücken aus der Orbita. Alle diese Erseheinungen traten zutage bei einem Rollenabstand von $18 \mathrm{~cm}$.

Experiment am 27, Angust 1908.

Hündchen 4, geboren in der Nacht vorn 16. auf den 17. August 1908. Gewicht $880 \mathrm{~g}$, Länge $36 \mathrm{~cm}$. Die Pupille reagiert auf Licht. Es wurde unterbunden, durchschnitten und gereizt der rechte Nervus vagosympathicus, wobei der gleiche Effekt erreicht wurde wie beim Hündchen III, 3.

\section{Wurf IV, bestehend aus sechs Jungen eines weiblichen Hofhundes.}

\section{Experiment am 28. Angust 1908.}

Hündehen 1, geboren am 24. August 1908. Gewicht $580 \mathrm{~g}$, Länge $17 \mathrm{~cm}$. Augen durch den Schnitt blossgelegt. Die Pupille reagiert nicht auf Licht. Es wurde unterbunden und durchschnitten der rechte Nervus vagosympathicus." Bei Reizung seines zentralen Stumpfes mittelst des elektrischen Stromes bei einem Rollenabstand von $15-10-5 \mathrm{~cm}$ trat Kontraktion der Nickhaut ein; alles andere blieb in statu quo.

\section{Experiment am 29. August 1908 (abends).}

Hündchen 2, geboren am 24. August 1908 um ca. 9 Uhr morgens. Gewicht $615 \mathrm{~g}$, Länge $19 \mathrm{~cm}$. Augen durch den Schnitt blossgelegt. Von seiten der Pupille ist die Reaktion auf Licht deutlich ausgesprochen. Die Pupille reagiert auch auf Schmerzreiz, der jedoch von bedeutender Intensität sein muss. Reizung des zentralen Stumpfes des durehschnittenen rechten Nervus vagosympathicus ruft Kontraktion der Nickhaut hervor; die Pupille jedoch bleibt unbeweglich.

Experiment am 30. August 1908.

Hündchen 3, geboren am 24. August 1908. Gewicht $680 \mathrm{~g}$, Länge $20 \mathrm{~cm}$." Augen durch den Schnitt blossgelegt. Die Pupille reagiert mit Verbreiterung auf Schmerz- und Verengerung auf Lichtreize. Der rechte Nervus vagosympathicus wurde unterbunden und durchschnitten, wobei Reizung seines zentralen Stumpfes mittelst des elektrischen Stromes bei einem Rollenabstand von 15-10 cm keinen Effekt seitens der Pupille nach sich zieht; es tritt aber eine Kontraktion der Nickhaut ein. 


\section{Experiment am 81. August 1908.}

Hündchen 4, geboren am 25. August 1908. Gewicht $720 \mathrm{~g}$, Länge $20 \mathrm{~cm}$. Augen durch den Schnitt blossgelegt. Die Pupille reagiert auf Licht- und auf Schmerzreiz. Aufsetzen der Elektroden auf den zentralen Stumpf des durchschnittenen rechten Nervus vagosympathicus ruft bei einem Rollenabstand von $15 \mathrm{~cm}$ energische Verbreiterung der Pupille und Kontraktion der Nickhaut hervor.

\section{Experiment am 1. September 1908.}

Hündchen 5, geboren am 24. August 1908. Gewicht $712 \mathrm{~g}$, Länge $21 \mathrm{~cm}$. Augen durch den Schnitt blossgelegt. Die Pupille reagiert auf Schmerz- und Lichtreiz. Es wurde unterbunden, durchschnitten und gereizt der rechte Nervus vagosympathicus, wobei bei einem Rollenabstand von $18 \mathrm{~cm}$ der gewöhnliche Effekt seitens der Pupille und der Nickhaut eintrat und auch Hervorrücken des Augapfels nach aussen beobachtet wurde.

\section{Experiment am 2. September 1908.}

Hündchen 6, geboren am 24. August 1908. Gewicht $730 \mathrm{~g}$, Länge $24 \mathrm{~cm}$. Augen durch den Schnitt blossgelegt. Die Pupille reagiert auf Licht und Schmerz; bei Reizung des Nervus vagosympathicus tritt der gleiche Effekt und unter den gleichen Bedingungen wie beim gestrigen Hündehen IV, 5 ein.

Ehe irgendwelche Schlussfolgerungen aus allen angeführten Tatsachen gezogen werden, halte ich es für zweckmässig, diese auf verschiedene Experimente verteilten Tatsachen in einer Tabelle zu gruppieren, in welcher das Zeichen $(+)$ bei dem Hündchen des gegebenen Alters die Anwesenheit der am Kopfende der betreffenden Spalte vermerkten Reaktion, das Zeichen (-) deren Abwesenheit bei dem betreffenden Hündchen angibt.

Alle von uns untersuchten Tiere wurden zum Experiment vor dem Eintritt der natürlichen Augenöffnung hinzugezogen; eine Ausnahme bildet in dieser Beziehung nur das Hündehen I, 5 (Experiment am 9. Juli 1908), welches schon ein Alter von 16 Tagen erreicht hatte, und bei dem zur Zeit des Experimentes die Augen schon auf naturlichem Wege geöffnet waren. 


\begin{tabular}{|c|c|c|c|c|c|c|}
\hline $\begin{array}{l}\text { Alter des } \\
\text { Hündchens }\end{array}$ & $\begin{array}{c}\text { Zeit } \\
\text { des Experi- } \\
\text { mentes } \\
1908\end{array}$ & $\begin{array}{l}\text { Reaktion } \\
\text { d. Pupille } \\
\text { auf Licht }\end{array}$ & $\begin{array}{c}\text { Reaktion } \\
\text { d. Pupille } \\
\text { auf } \\
\text { Schmerz }\end{array}$ & $\begin{array}{c}\text { Reaktion } \\
\text { der Pupille } \\
\text { auf Reizung } \\
\text { des N. vago- } \\
\text { symp. }\end{array}$ & $\begin{array}{c}\text { Reaktion } \\
\text { der Niekhaut } \\
\text { auf Reizung } \\
\text { des N. vago- } \\
\text { symp, }\end{array}$ & $\begin{array}{l}\text { Bewegung } \\
\text { des Aug- } \\
\text { apfels nach } \\
\text { aussen bei } \\
\text { Reizung des } \\
\text { N.vagosymp. }\end{array}$ \\
\hline 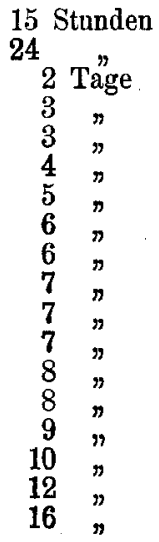 & 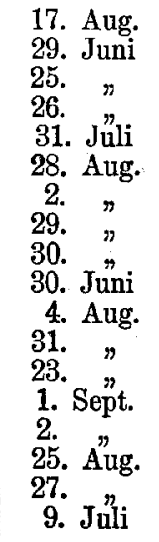 & $\begin{array}{l}- \\
= \\
= \\
= \\
= \\
+ \\
+ \\
+ \\
+ \\
+ \\
+ \\
+ \\
+ \\
+\end{array}$ & $\begin{array}{l}+ \\
+ \\
+\end{array}$ & $\begin{array}{l}- \\
z \\
z \\
= \\
= \\
\bar{t} \\
+ \\
+ \\
+ \\
+ \\
+ \\
+ \\
+\end{array}$ & $\begin{array}{l}- \\
- \\
+ \\
+ \\
+ \\
+ \\
+ \\
+ \\
+ \\
+ \\
+ \\
+ \\
+ \\
+ \\
+ \\
+\end{array}$ & $\begin{array}{l}- \\
= \\
= \\
= \\
= \\
- \\
+ \\
+ \\
+ \\
+ \\
+ \\
+\end{array}$ \\
\hline
\end{tabular}

Diese Tabelle, die auf Grund der obenangeführten Tatsachen zusammengestellt ist, zeigt, dass:

1. bei jungen Hunden, die, wie bekannt, mit geschlossenen Augenzur Welt kommen, der Pupillar-und andere Augenreflexe nicht in fertiger und vollentwickelter Weise schon bei der Geburt präexistieren, sondern erst in den ersten Tagen des extrauterinen Lebens zur Entwicklung gelangen, wobei sienatürlich als vererbte aufzufassen sind;

2. zurZeit der natürlichen Augenöfnung die neugeborenen Tiere schon über alle Pupillar-Augenreflexe in wohlentwickelter Form verfügen;

3. verschiedenedieser Reflexe sich in verschiedener Frist nach der Geburt des Tieres entwickeln;

4. früher als die anderender Reflex der Nickhautkontraktion bei Reizung des Nervus vagosympathicus zur Entwicklung kommt-nach Erreichung des Alters von 3 Tagen;

5. nach 5 Tagen die Pupille auf Lichtreiz zu reagieren beginnt;

6. nach 5 Tagen auch die Reaktion der Pupille auf Schmerzreiz bedeutender Intensität deutlich ausgesprochen ist; 
7. der gewöhnliche durch Reizung des Nervus vagosympathicus bedingte Pupillarreflex zum erstenmal auftritt, wenn das Tier das Alter von 7 Tagen erreicht hat;

8. wenn das Tierdas Alter von 8 , mitunter auchnur von 7 Tagen erreicht hat, bei Reizung des Nervus vago sympathicus Hervorrücken des Augapfels nach aussen eintritt;

9. der Pupillar-und die anderen Augenreflexesich entwickeln, folglich um einige Tage früher, als ihr Vorhandensein fürden $0 \mathrm{rganismus}$ in dieser oderjener Lebenslage notweidig erseheinen kỏnnte.

Einige dieser Sehlussfolgerungen decken sich mit manchen klinischen Tatsachen und Beobachtungen; beziehentlich des neugeborenen Kindes jedoeh will ich mich mit der Aufstellung: solcher Análogien absolut nicht abgeben, weil ja das Kind mit offenen Augen zur Welt kommt und sich folglich in der Hinsicht unter anderen Bedingungen befindet als der neugeborene Hund.

Um die Untersuchung der Pupillar- und Augenreflexe auch auf solche Tiere auszudehnen, die mit offenen Augen zur Welt kommen, wurden Experimente am Meerschweinchen angestellt.

Experiment am 13. November 1908.

Meerschweinchen, geboren um 8 Uhr morgens des 13 . November 1908. Zur Zeit des Experimentes war es 2 Stunden und einige Minuten alt. Zweeks besserer Besichtigung des Auges wurden die oberen sowohl als die unteren Augenlider abgeschnitten, nachdem festgestellt worden war, dass die Pupille deutlich auf Lichtreiz reagiert. Darauf wurde unterbunden und durchschnitten der cervicale Nervus sympathicus; auf Reizung seines zentralen Stumpfes mittelst des elektrischen Stromes von der Sekundärspirale eines DuboisReymond'schen Apparates beim Rollenabstand von $18 \mathrm{~cm}$ traten Verbreiterung der Pupille, Kontraktion der Nickhaut und Hervorrücken des Augapfels nach aussen ein.

Experiment am 17. November 1908.

Meerschweinschen, geboren in der Nacht vom 16. auf den 17. November 1908. Von der Geburt an sieht und läuft es. Bei Reizung des zentralen Stumpfes des durchschnittenen Nervis 
sympathicus am Halse trat der gewöhnliche Oculopupillarreflex, wie er auch beim erwachsenen Tier beobachtet wird, ein. Fin zweites Meerschweinchen derselben Nummer gab unter den gleichen Bedingungen genau die gleichen Resultate. Dasselbe wurde bei einem Meersehweinchen, das am 13. November 1908 um 8 Uhr morgens zur Welt kam und folglich das Alter von 4 Tagen erreicht hatte festgestellt.

Auf Grund dieser Tatsachen kommen wir zum Schlusse, dass :

1. neugeborene Meerschweinchen, die bekanntlich mit offenen Augen zur Welt kommen, gleich von der Geburt an über wohlentwickelte oculo-pupilläre Reflexe verfügen;

2. diese Reflexe sich wahrscheinlich in den letzten Tagen des intrauterinen Lebens entwickeln.

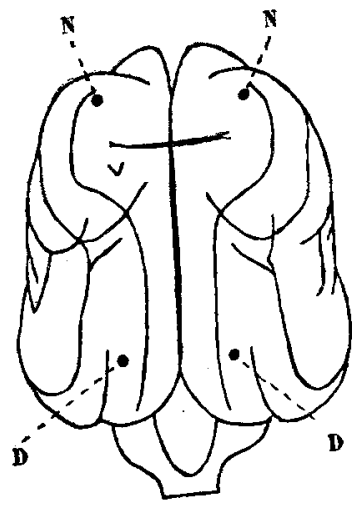

Fig. 1.

Bevor ich die vorliegende Arbeit schliesse, will ich noch kurz Auszüge aus jener Kategorie von Experimenten anführen, welche zum Studium der Erregbarkeit der motorischen Rindenzentren angestellt wurden, und bei welehem es gelang unter anderem auch die Verbreiterung der Pupille bei Reizung bestimmter Punkte zu beobachten. Zu den genannten Experimenten dienten neugeborene Hunde (s. die entsprechende Arbeit Pflüg er's Archiv):

Aus diesen Experimenten folgt, dass es bei Reizung der Hirnrinde neugeborener Hunde mittelst des elektrischen Stromes bis zum Alter von 9 Tagen nicht gelingt, als motorischen Effekt dieser Reizung eine Verbreiterung der Pupille zu bekommen. Von dem genannten Alter an aber tritt Verbreiterung der Pupille bei Reizung des Punktes $D$ (Fig. 1) im Occipital-und des Punktes $N$ (Fig. 1) im Frontalgebiet ein. Ausführlichere Angaben finden sich in der Arbeit "Zur Frage über die Erregbarkeit der motorisehen Zentra in der. Hirnrinde neugeborener Säugetiere". (Pflüger's Arch. f. d. ges. Physiol. Bd. 133 S. 45. 1910.)

Das auf Fig. 1 abgebildete Gehirnschema stammt von einem sechs Tage alten Hündchen. 